\title{
Zur Psychiatrie von Corona
}

\author{
Klinischer Alltag, Psychopathologie, Long-Covid, Impfen, \\ Demonstrationen und gedrückte Stimmung
}

Die Lage der Welt ist derzeit bestimmt durch eine globale Pandemie, die zwar vor allem den Respirationstrakt betrifft, aber auch die Psychiater und Neurologen beschäftigt. Denn der Erreger SARS-CoV-2 befällt auch Leber, Nieren, Pankreas und das Gehirn, wie man schon seit Sommer 2020 nicht zuletzt aufgrund von Autopsien weiß $[4,8,19]$. Diese hatten aber auch gezeigt, dass das Virus nur in 20-30\% der Fälle überhaupt im Gehirn nachweisbar war, was nicht zur klinischen Häufigkeit von Zeichen der ZNS-Beteiligung (Müdigkeit, Abgeschlagenheit, Geruchsstörungen) von bis zu $85 \%$ bei symptomatischen Patienten passte. Eine kürzlich im Fachblatt Nature Medicine publizierte Studie konnte jedoch im Mausmodell mit Hilfe der radioaktiv markierten S1-Untereinheit des Coronavirus-Spike-Proteins, das intravenös verabreicht worden war, dessen Gehirngängigkeit nachweisen. Im Gegensatz zum Virus passiert dieses Protein also die Blut-HirnSchranke und findet sich in allen 11 untersuchten Regionen des Gehirns, wo es toxische Effekte haben und funktionseinschränkend wirken kann. Die Autoren schreiben: „Coronavirus-Spike-Proteine werden oft durch Proteasen der Wirtszelle vom Virus abgespalten. Einmal gespalten, sind die Coronavirus-Spike-Untereinheiten S1 und S2 nicht mehr kovalent durch Disulfid-Brücken gehalten, sodass S1 sich von den Viruspartikeln trennt. Es besteht daher die Möglichkeit, dass S1-Untereinheiten während einer Infektion mit SARS-CoV-2 die BlutHirn-Schranke überwinden und im Gehirn pathologische Reaktionen auslösen, ohne dass intakte Viruspartikel ins Gehirn gelangt sind“ [11].

\section{Psychopathologie}

„Jede Grippe macht einen matt und schlapp, Corona auch und ist daher nicht anders zu beurteilen. “ - So oder so ähnlich denken noch immer viele Menschen über das Virus SARS-CoV-2 und die dadurch ver- ursachte Erkrankung Covid-19. Diese Meinung hat sich längst als falsch herausgestellt, sofern man den Anteil der schweren Verläufe, die weit über eine Lungenentzündung hinausgehende Pathologie und die vergleichsweise deutlich höhere Letalität berücksichtigt. Aber auch bei der Psychopathologie gibt es klare Unterschiede. Diese dingfest zu machen, ist jedoch gar nicht so einfach, denn bei fast allen Studien zu den psychiatrischen Folgen von Covid-19 handelte es sich um Umfragen zu selbstberichteten Symptomen, nicht jedoch um Diagnosen. Angst und Deprimiertheit wurden auf diese Weise zwar gefunden, jedoch eher qualitativ, und nur selten fanden sich Hinweise auf das Risiko einer Demenz als mögliche Folge von Covid-19. Hierzu bedarf es quantitativer Studien zu faktisch vorliegenden Diagnosen.

Die bislang größte Studie hierzu erschien im Fachblatt Lancet Psychiatry. Insgesamt fast 70 Millionen elektronische Krankenakten wurden nach Fällen von Covid-19 im Zeitraum vom 20.1.2020 bis 1.8.2020 durchsucht. Die gefundenen 62354 Fälle wurden im Hinblick auf neuropsychiatrischen Folgen - Ängste, Depression, Demenz, Insomnie - im Zeitraum von 14-90 Tagen nach Symptombeginn von Covid-19 mit den gleichen Folgen von anderen häufigen Erkrankungen (Influenza, Gallenblasen-OP, Knochenbruch) in dieser Datenbank während des genannten Beobachtungszeitraums verglichen [21]. Dies macht den besonderen Charme der Studie aus: Man erhielt das relative Risiko beispielsweise einer Angststörung im Zeitraum von 2 Wochen bis 3 Monaten nach dem Beginn einer Covid-19-Erkrankung im Vergleich zu den Folgeerscheinungen nach einer Grippe (Influenza), einer Gallen-OP oder einem Knochenbruch. Diese „Kontrollbedingungen“ können aus verschiedenen Gründen ebenfalls zu psychologischen Beeinträchtigungen führen, weswegen ein Vergleich mit ihnen das spezifische Risiko von Covid-19 für die Entwicklung psychiatrischer Erkrankungen herausarbeitet.

Bei Patienten mit „Zustand nach Covid19“ und keinen psychiatrischen Vorerkrankungen war das Risiko einer psychiatrischen Erstdiagnose im Vergleich zu „Zustand nach Influenza“ um den Faktor 2,1 höher. Auch verglichen mit „Zustand nach Gallenstein-OP“ und „Zustand nach Knochenbruch" hatte eine abgelaufene Covid-19-Erkrankung ein entsprechendes auf das 2,2- bzw. 2,1-fach erhöhtes Risiko. Die Inzidenz irgendeiner psychiatrischen Diagnose nach abgelaufener Covid-19-Erkrankung betrug 18,1\%. Die häufigste psychiatrische Diagnose nach Covid-19 war mit 12,8\% die Angststörung, gefolgt von affektiven Störungen (hauptsächlich Depression) mit 9,9\%. Bei den über 65-Jährigen war das Risiko, im genannten Zeitraum nach einer Covid-19-Erkrankung an Demenz zu erkranken, um $65 \%$ erhöht, ein besonders wichtiges Ergebnis, das nur aufgrund der hohen Fallzahl überhaupt gefunden werden konnte, wie die Autoren hervorheben. Auch die Wahrscheinlichkeit des Auftretens von Schlafstörungen ist nach abgelaufener Covid-19-Erkrankung nahezu verdoppelt [21]. Die Autoren gingen darüber hinaus der Frage nach dem umgekehrten Zusammenhang - steigt das Risiko, an Covid-19 zu erkranken, wenn eine psychiatrische Erkrankung im Jahr davor bereits vorlag? - nach und fanden tatsächlich einen entsprechenden Zusammenhang: Die Wahrscheinlichkeit, an Covid-19 zu erkranken, ist um $65 \%$ erhöht. Allerdings geben die Autoren zu bedenken, dass dieser Effekt auf das Konto des sozioökonomischen Status geht, denn ein geringer sozioökonomischer Status prädisponiert sowohl für Covid-19 als auch für psychiatrische Erkrankungen.

Angesichts dieser Daten wundert es nicht, dass im klinischen Alltag auch in der Psychiatrie Ulm psychiatrische Konsequenzen bzw. coronabedingte Psychopatholo- 
gie eine Rolle spielen. Das Problem dabei ist, dass SARS-CoV-2-positive psychisch kranke Menschen für alle an der Behandlung beteiligten Berufsgruppen einen deutlichen Mehraufwand bedeuten ( $\triangleright$ Abb. 1). Der Schutz vor Ansteckung und potenzieller Ausbreitung von SARS-CoV-2 über das Personal auf andere Patienten hat oberste Priorität und verlangt allen Mitarbeitern sehr viel ab. Insbesondere für das Pflegepersonal bedeutet jeder Covid-19-Patient einen erheblichen zusätzlichen Zeitaufwand. Psychologen behandeln entweder über das Telefon, einen Video-Chat (Zoom etc.) oder aber auch vor Ort - dann selbstverständlich ebenfalls in „voller Montur“. Kotherapien finden schon lange nicht mehr in Gruppen von bis zu 6 Patienten statt, sondern meist einzeln. Auch dies bedeutet einen erheblichen Mehraufwand bei gleichzeitig geringerem Angebot. Da die Pflege einen Teil der Tagesstrukturierung mit übernehmen muss, ist sie gleich doppelt belastet. Vieles geht über das Telefon und dauert nicht zuletzt genau deswegen einfach länger (z. B. die in der Psychiatrie extrem wichtige Sozialarbeit). Besuche durch Angehörige und Gespräche mit Angehörigen werden zum Problem, weil durch sie ein zusätzliches Risiko der Ansteckung besteht. Über Weihnachten werden an Besucher, die ihren Mund-Nasen-Schutz vergessen haben, vom Universitätsklinikum Ulm FFP2-Masken zur Verfügung gestellt. Keiner soll wegen einer vergessenen Maske seinen Angehörigen nicht besuchen können!

Seit Mitte März 2020 treffen wir uns zur Frühbesprechung mit Maske ( $\triangleright$ Abb. 2), was zunächst sehr gewöhnungsbedürftig war: Man hört die Sprache und sieht die Emotionen der Mitarbeiter deutlich schlechter, muss daher mehr nachfragen und Emotionen zuweilen explizit verbal kommunizieren, weil es implizit nonverbal nicht mehr geht [18].

Kurz: Die Corona-Pandemie stellt für das gesamte System der Versorgung psychisch kranker Menschen eine Herausforderung dar: Es gibt zum einen coronabedingt mehr Fälle und zum zweiten ist die Versorgung aller Fälle wegen der Ansteckungsgefahr erheblich schwieriger und vor allem zeitaufwändiger geworden. Hinzu kommen die Notwendigkeit der Distanzierung, der Ausfall von Gruppen und die dadurch gerin-

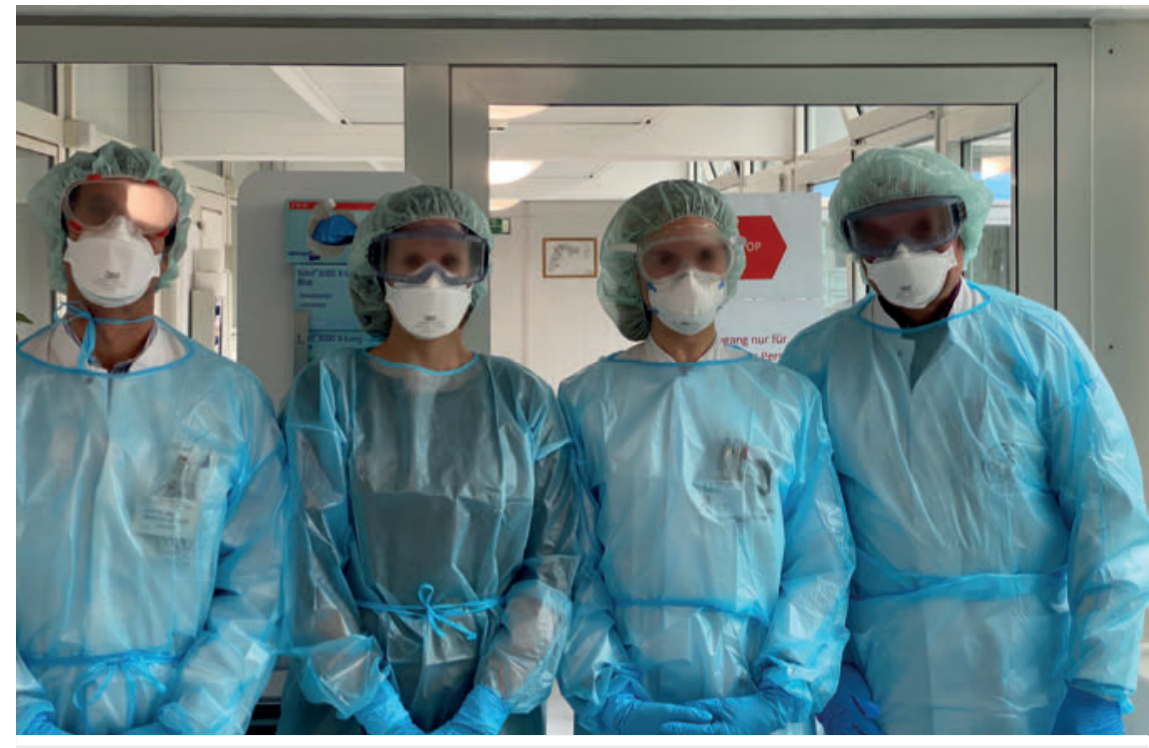

Abb. 1 Chefvisite durch den Autor (ganz rechts) und 3 Mitarbeiter in Zeiten von Corona (c) Autor).

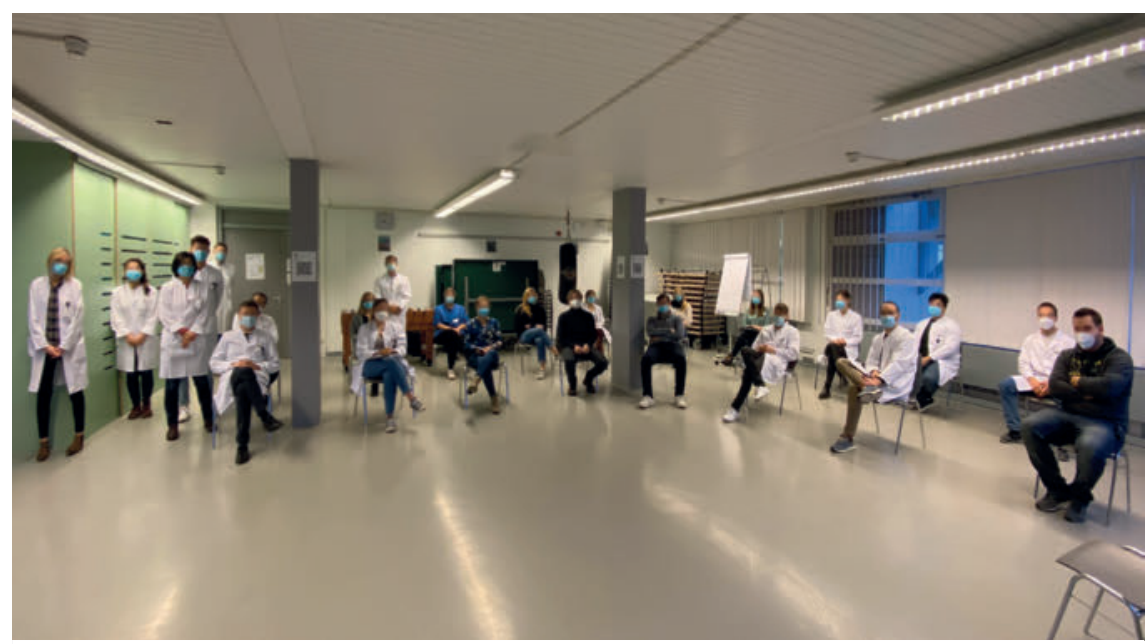

- Abb. 2 Frühbesprechung mit Abstand und Maske aus der Sicht des Chefs (৫) Autor).

gere Effektivität von Mitarbeitern. Wenn sie dann noch wegbleiben würden [13] würde das System versagen. Dass es hier bei uns noch nicht soweit gekommen ist, verdanke ich meinen Mitarbeitern, die ich nach Kräften über alles Neue und Wissenswerte zu Corona und der Pandemie informiere (was sie dann mit 2 Tagen Verspätung auch aus der Tagesschau erfahren), sodass sie alles wissen, was ich auch weiß - und auch das wissen sie. Das ist eine der bekannten Voraussetzungen dafür, dass Mitarbeiter nicht aufgeben und motiviert mitmachen. Geheimniskrämerei und Befehlston von oben waren noch nie Zeichen eines guten Führungsstils. In einer Pandemie sind sie absolut kontraindiziert.

\section{Long-Covid}

Wie eingangs erwähnt, war das Ausmaß der Bedrohungen durch das neue Coronavirus SARS-CoV-2 zu Beginn der Pandemie im Frühjahr noch nicht klar. Hinzu kam die Erkenntnis, dass die Krankheit langfristig bestehen bleiben kann - man spricht von „Long-Covid“, post-Covid-19 syndrome, chronic Covid syndrome (CCS), long-haul Covid und chronic Covid. Erste Berichte hierüber lagen bereits im Sommer vor, jedoch wurden erst im Herbst das ganze Ausmaß und die Chronizität der Beeinträchtigung sichtbar. Schon am 8. April 2020 wurde im Fachblatt Science die Vermutung geäußert, dass das Überstehen der Akutphase 
von Covid-19 für viele Patienten nur der Anfang einer länger währenden Erkrankung darstellen könnte [12]. Eine seither zunehmende Zahl von Studien zeigt genau dies überdeutlich. Am 9. Juli publizierten italienische Ärzte im Fachblatt JAMA eine Studie an 143 Patienten, von denen im Mittel 60 Tage nach dem Beginn der Symptome von Covid-19 noch 87,4\% mindestens ein Symptom aufwiesen [2]. Die meisten klagten über Abgeschlagenheit („fatigue“) und Atembeschwerden („dyspnea“). Die Patienten waren im Mittel 56,5 Jahre alt, 37\% waren weiblich. Während ihres im Mittel 13,5-tägigen Krankenhausaufenthalts hatten $72,7 \%$ von ihnen eine interstitielle Pneumonie, nur 7 Patienten ( $5 \%$ ) waren invasiv beatmet worden.

Am 14. Oktober publizierten britische Autoren eine prospektive Längsschnittstudie an 201 Patienten mit abgelaufener Covid-19-Erkrankung (mittleres Alter: 44 Jahre; $70 \%$ weiblich) und anhaltenden Symptomen. Die Patienten waren zwischen April und September 2020 für im Mittel weitere 105-160 Tage (Median: 140 Tage) nachbeobachtet worden. Die Prävalenz von Vorerkrankungen (Adipositas: $20 \%$, Bluthochdruck: $6 \%$; Diabetes: $2 \%$; Herzerkrankungen: $4 \%$ ) war insgesamt gering, und nur $18 \%$ der Patienten mussten stationär behandelt werden. Dennoch bestand gut viereinhalb Monate nach dem ersten Auftreten der Symptome bei nahezu allen Patienten noch Müdigkeit (98\%); Muskelschmerzen (88\%), Kurzatmigkeit (87\%) und Kopfschmerzen (83\%) waren ebenfalls noch sehr häufig vorliegende Symptome und $42 \%$ (!) der Patienten litten noch unter 10 oder mehr (!) Symptomen. Damit hatten in einer vergleichsweise jungen und risikoarmen Population mit anhaltenden Symptomen fast $70 \%$ der Personen 4 Monate nach den ersten Symptomen einer SARS-CoV-2-Infektion eine Beeinträchtigung in einem oder mehreren Organen: Herz (32\%), Lunge (33\%), Nieren (12\%), Leber (10\%), Pankreas (17\%) und Milz (6\%). Bei $66 \%$ der Patienten wurden Beeinträchtigungen einzelner Organe und bei $25 \%$ der Patienten Beeinträchtigungen mehrerer Organe beobachtet ( $\vee$ Abb. 3).

Am 9. November veröffentlichte ein irisches Autorenteam Daten zu 128 Patienten (mittleres Alter 49,5 Jahre, $54 \%$ weiblich) im Mit-

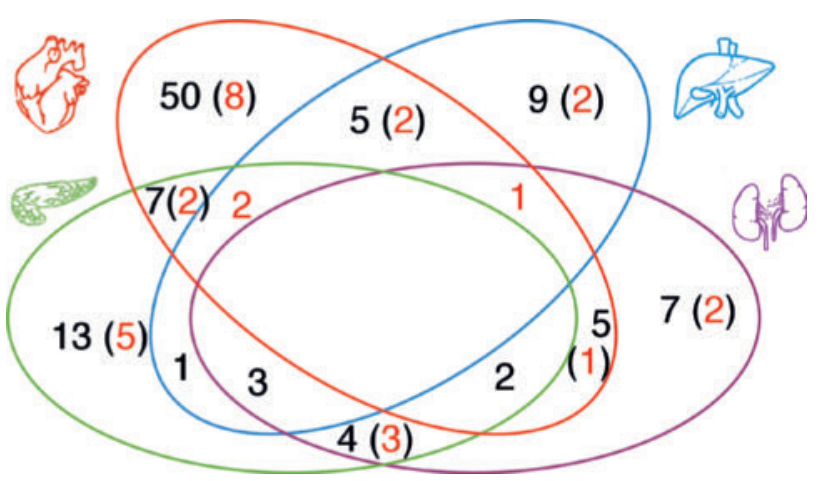

Abb. 3 Organbeteiligung von Herz, Leber, Pancreas und Nieren, allein bzw. in unterschiedlichen Kombinationen bei insgesamt 201 Patienten mit geringem Risiko (nur 37 davon waren hospitalisiert, rote Zahlen beziehen sich auf diese Untergruppe) aber mit Long-Covid (nach Daten aus [29]).

tel 70 Tage nach Beginn der Covid-19-Symptome, von denen mehr als die Hälfte über anhaltende Müdigkeit (67/128; 52,3\%) berichteten. Interessanterweise gab es keinen Zusammenhang zwischen dem Schweregrad der anfänglichen Covid-19-Erkrankung (stationäre Aufnahme oder nicht, zusätzliche Sauerstoffzufuhr oder nicht) und der Müdigkeit später. Zudem wurden auch keine statistischen Zusammenhänge zwischen der Müdigkeit und Routinelaborparametern für Entzündung und Zellumsatz (Leukozyten-, Neutrophilen- oder Lymphozytenzahl, Neutrophilen-Lymphozyten-Verhältnis, Laktat Dehydrogenase, C-reaktives Protein) bzw. proinflammatorischen Molekülen (IL-6, sCD25) gefunden. Weibliches Geschlecht und das Vorbestehen einer Depression scheint jedoch nach diesen Daten ein Risikofaktor für Fatigue zu sein. Die Autoren heben hervor, dass diese Gruppe von Patienten bislang verglichen mit den akuten Fällen noch zu wenig Beachtung findet [23].

Ging man bei Covid-19 (wie auch bei vielen anderen Virusinfektionen beispielsweise der Infektiösen Mononukleose) bislang von einem geringen Risiko bei jungen Menschen ohne Komorbiditäten aus, sprechen die hier vorgestellten Daten eher für einen protrahierten Verlauf - auch nach nur leichten Symptomen. Dies hat nicht nur Auswirkungen auf die Belastung einzelner Patienten durch lang andauernde Covid-19-Symptome, sondern ist auch unter Public-Health-Gesichtspunkten von Bedeutung. Auch bei der Infektiösen Mo- nonukleose (Epstein-Bar-Virus) kommt Fatigue 6 Monate nach der Infektion bei 40\% der Patienten vor [26], man folgte jedoch bislang dem Rat der $\mathrm{WHO}^{1}$, die Patienten bei komplikationslosem Verlauf generell 4 Wochen nach Erkrankungsbeginn wieder für arbeitsfähig zu erklären. Nach den Daten zu Long-Covid muss dies in Frage gestellt werden. Im bisherigen Verlauf der Corona-Pandemie kam es zunächst zu Einbrüchen der Belegung in Reha-Kliniken. Mittlerweile muss man jedoch davon ausgehen, dass mittelfristig ein erhöhter Bedarf an Rehabilitationsmaßnahmen bestehen wird, weil die Gruppe der Long-Covid-Patienten zu den üblichen Reha-Patienten (nach Schlaganfall, Herzinfarkt, Bandscheibenvorfall etc.) noch hinzukommen wird, wovon mittlerweile Experten aus Neurologie, Psychiatrie, Innerer Medizin, Virologie, Psychosomatik, Kardiologie und Intensivmedizin ausgehen. Im deutschen Gesundheitssystem wird man mit den Langzeitfolgen der Covid-19-Pandemie noch lernen müssen umzugehen.

1 „General practitioners and occupational physicians should avoid advising rest, in order to prevent prolonged fatigue and failure to recover. If there are no complications, it is safe to start gradual return to work 4 weeks after the onset of infectious mononucleosis“ ([6]; zu Deutsch: „Allgemeinmediziner und Arbeitsmediziner sollten nicht Ruhe verordnen, um anhaltende Müdigkeit und ausbleibende Genesung zu verhindern. Bei komplikationslosem Verlauf kann 4 Wochen nach Beginn der infektiösen Mononukleose mit der schrittweisen Rückkehr zur Arbeit begonnen werden“). 


\section{Impfen}

Herkömmliche Impfstoffe gegen Viren enthalten ein Protein aus der Hülle des Virus (in irgendeiner Form). Je älter die Impfstoffe sind, desto kruder die Verfahren der Herstellung: Viren können mit Hitze oder auf chemischem Wege so verändert werden, dass sie nicht mehr infektiös sind, aber ihre Bestandteile noch eine Immunreaktion hervorrufen. In solchen Impfstoffen sind somit sehr unterschiedliche Stoffe enthalten (weswegen sie zuweilen auch als gewissermaßen sehr „dreckig“ bezeichnet werden). Mehrere der neu entwickelten Impfstoffe gegen Covid-19 sind dagegen mRNA-Impfstoffe, die nur den Botenstoff (messenger Ribonucleic Acid; mRNA; zu deutsch mRNS, d. h. „Säure“ für „acid) für ein einziges Protein enthalten (und daher mit gutem Recht als vergleichsweise „sauber" bezeichnet werden können). Dies trifft für die Impfstoffe BNT162b2 der Firmen BioNtech (Mainz) und Pfizer (USA), mRNA-1273 der Firma Moderna (USA) und CVnCoV der Firma CureVac (Tübingen) zu. ${ }^{2}$ Messenger-RNA (mRNA) für ein Virusprotein, die in die Zellen des Patienten gelangt, setzt dort eine Übersetzung (Translation) der Information zum Aufbau des Proteins in Gang, die das Protein erzeugt. Dieses gelangt an die Zelloberfläche, hat Kontakt mit dem Immunsystem und wirkt damit als Antigen, gegen das eine Immunreaktion gestartet wird. Das Immunsystem lernt im Verlauf dieser Immunreaktion, selektiv Zellen zu bekämpfen, die solche Antigene auf ihrer Zelloberfläche tragen, wie beispielsweise durch Viren infizierte Zellen. Das Verfahren ist bereits 30 Jahre alt, denn die Herstellung von RNA außerhalb eines Organismus (in vitro) mit anschließender Translation in einem Organismus (in vivo) wurde erstmals bereits im Jahr 1990 beschrieben [28]. Im Jahr 1994 wurde RNA erstmals zur Impfung bei Mäusen verwendet [30].

Die RNA selbst erzeugt dabei keine Immunreaktion und wird nach kurzer Zeit in der Zelle abgebaut. Hierzu eine Anmerkung: Botenstoffe müssen grundsätzlich relativ schnell abgebaut werden, sonst könnten sie nicht die Eigenschaft eines Botens haben:

2 Eine gute Übersicht zu den neuesten Entwicklungen in Deutschland (Stand: 19.12.2020) gibt der Verband Die forschenden Pharma-Unternehmen (vfa) [24].
Verblieben sie lange in den Zellen, hätte ihre Präsenz nicht mehr den Charakter einer Nachricht - ganz prinzipiell. Ebenso können mRNA-Impfstoffe prinzipiell gegen alle proteinbasierten Antigene entwickelt werden, also beispielsweise von Viren und Bakterien, aber auch von Tumoren. Aus genau diesem Grund wird seit Jahren sehr intensiv an ihnen gearbeitet, und möglicherweise wird es bald nur noch solche Impfstoffe geben. Probleme liegen wie immer im Detail. Beispielsweise müssen die als Antigen wirkenden Bereiche eines Proteins (dessen „Epitope“) an einen Haupthistokompatibilitätskomplex (MHC mit der Nummer I) gekoppelt sein, sonst klappt die zelluläre Immunantwort nicht. Die humorale Immunantwort (Antikörper) hingegen bedarf des Haupthistokompatibilitätskomplexes MHC mit der Nummer II. Man braucht also jede Menge Detailkenntnisse über die bei Immunreaktionen beteiligten Mechanismen. Ein ganz anderes Detailproblem besteht darin, die RNA überhaupt erst in die Zellen des Körpers zu verbringen [9]. Sie einfach frei in Ringerlösung zu injizieren funktioniert nicht, weil sie nicht von den Zellen aufgenommen wird. Hierzu wird die RNA in winzig kleine (80 nanometer Durchmesser) Lipid-Membran-Kügelchen „verpackt“ und eine Emulsion dieser Fettkügelchen wird injiziert.

Bekanntermaßen erhielt der Impfstoff BNT162b2 der Firmen BioNtech und Pfizer in den USA und in Großbritannien aufgrund der positiven Erfahrungen in der Phase-III-Studie an 43448 Probanden, von denen 21720 den Impfstoff und 21728 ein Placebo injiziert bekamen, eine Notzulassung. Diese Studie wurde im New England Journal of Medicine am 10.12.2020 online publiziert [10]. Bereits einen Tag später - am 11.12.2020 - erteilte die US-amerikanische Gesundheitsbehörde FDA, die in den Monaten und Wochen zuvor alle relevanten Informationen bereits jeweils unmittelbar nach deren Generierung erhalten hatte, eine Notfallzulassung des Impfstoffs [7]. Warum die entsprechende Behörde der EU daraufhin bekannt gab, dass auch sie Ende des Jahres eine reguläre Zulassung vielleicht würde erteilen können, ist angesichts der Dringlichkeit der Lage - bis zu 30000 Neuinfizierte täglich allein in Deutschland, in der gesamten EU sehr hohe Fallzahlen - aus meiner Sicht nicht nachvollziehbar. ${ }^{3}$

Bislang weiß niemand genau, wie lange eine durch Impfen hervorgerufenen Immunität anhalten wird. Es gibt jedoch erste Daten hierzu, selbst wenn es noch keine Daten zur Immunität nach Impfen geben kann, da mit dem Impfen erst Ende 2020 begonnen wurde. In einer im Fachblatt Science publizierten Untersuchung US-amerikanischer Autoren wurde in einer Kohorte von 30082 infizierten Patienten mit leichten bis mittelschweren Covid-19-Symptomen die Robustheit und Langlebigkeit der Anti-SARS-CoV-2-Antikörperantwort gemessen [25]. Sie fanden heraus, dass neutralisierende lgG Antikörpertiter gegen das SARS-CoV-2-Spike-Protein für mindestens 5 Monate nach der Infektion persistieren. Man wird diese Kohorte natürlich weiter untersuchen, um die Langlebigkeit und Stärke dieser Reaktion zu messen. Dennoch deuten diese vorläufigen Ergebnisse darauf hin, dass die Wahrscheinlichkeit einer Reinfektion geringer sein könnte, als derzeit befürchtet wird, und dass für eine Impfung die generell für Impfungen von der WHO geforderte Mindestwirksamkeitsdauer von einem halben Jahr gegeben ist. Zu einem ähnlichen Ergebnis kam auch eine kleine Studie aus Innsbruck [3].

\section{Demonstranten, Covid-Leugner und Verschwörungsanhänger}

Konnte man im vergangenen März noch behaupten, das neue Coronavirus sei gar nicht so schlimm oder existiere nicht wirklich oder nur als einfaches Erkältungs- und Grippevirus, so geht dies zum Jahresende einfach nicht mehr: Weltweit mehr als 75 Millionen Infizierte und mehr als 1,7 Millionen Tote kann man ebenso wenig leug-

3 Die offizielle Begründung lautete, man wolle keine Notfallzulassung, sondern eine „richtige“ Zulassung. Aber aufgrund welcher zusätzlichen Daten sollte die denn erfolgen? Es gibt ja keine wirklich neuen Daten, sieht man einmal von einigen wenigen seltenen Zwischenfällen ab, die keinerlei allgemeine Erkenntnisse ergeben. Und ein „Notfall“ lag ja definitiv vor, sonst hätte man die vielen Maßnahmen der Einschränkung (Lockdown) nicht verordnet. 
nen wie die Übersterblichkeit in fast allen Ländern (einschließlich Deutschland), die zunehmende Aus- und drohende Überlastung der Intensivkapazitäten, auch in Deutschland, und die ans Chaotische grenzenden Zustände im reichsten und zugleich noch vor einem Jahr als am besten von allen Ländern der Erde auf eine Pandemie vorbereitesten Land, den USA. Dort gibt es über 17 Millionen Infizierte und mehr als 300000 Tote. Allein in den letzten 14 Tagen (2. bis 15. Dezember) betrug die Zahl der Corona-Neuerkrankungen dort 2973498. Zum Vergleich: Die Zahl der Neuerkrankungen in diesem Zeitraum wurde auch in Deutschland als dramatisch erlebt, betrug aber nur etwa ein Zehntel des Wertes aus den USA bei etwa einem Viertel der Einwohnerzahl. Pro Kopf erkrankten in den USA in der ersten Dezemberhälfte also etwa 2,5mal mehr Menschen als hierzulande. Wie kann man angesichts dieser Tatsachen in Deutschland gegen Corona demonstrieren oder die Krankheit gar leugnen? Was treibt die Menschen dazu, an abstruse Theorien (Bill Gates und/oder Angela Merkel wollen die Demokratie abschaffen etc.) zu glauben und sich auch so zu verhalten [14]?

Aus meiner Erfahrung zu Versuchen der Diskussion mit Corona-Leugnern kann ich dazu nur sagen, dass diese den Gesprächen mit Wahnkranken sehr ähneln: Man kann sagen, was man will, das Gegenüber bleibt bei seiner Version der Welt mit subjektiver Gewissheit und vollkommener Unkorrigierbarkeit der Inhalte. Es wird ausgewichen, Fakten werden einfach geleugnet und vage Vermutungen über böse Mächte, Lügen und eine drohende Gefahr werden immer wieder geäußert. Man weiß, dass solches Erleben und Verhalten bei Psychosen vorkommen, aber auch durch Isolation und mangelnde Kommunikation (z. B. bei Schwerhörigen oder bei Menschen, die sich allein in sprachfremder Umgebung befinden) vorkommen kann. Man weiß auch, dass Verschwörungstheorien von existenziell benachteiligten Menschen bzw. Menschen, die den Verlust der Kontrolle über ihr Leben erfahren, mit höherer Wahrscheinlichkeit geäußert werden. Und man weiß schließlich, dass Psychosen häufig sind und dass es ein Kontinuum gibt zwischen psychischer Gesundheit, schizotypischer Persönlichkeit, schizotyper Persönlichkeitsstörung und Schizophrenie. Aus meiner Sicht

- Tab. 1 Namen und Daten der Entstehung von Webseiten zum (a) Lockdown-Protest, (b) Betrug und (c) gesundheitsbezogener Falschinformation (nach Daten aus [1]). Jeweils 3 von 40 Webseiten sind beispielhaft aufgeführt.

\begin{tabular}{|l|l|l|}
\hline \multirow{2}{*}{ Typ } & Name & Online seit... \\
\hline \multirow{2}{*}{ Lockdown Protest } & reopenarizona.com & 9.4 .2020 \\
\hline \multirow{2}{*}{ Betrug } & liberateamericanow.com & 24.4 .2020 \\
\hline & letamericaopen.net & 2.5 .2020 \\
\hline gesundheitsbezogene & annaasiasurgicalmasks.com & 11.4 .2020 \\
\hline Falschinformationen & theluxuryhealing.com & 1.5 .2020 \\
\hline & tropicalprotectivewear.com & 16.8 .2020 \\
\hline & fakepandemic.com & 23.3 .2020 \\
\hline & humansarefree.com & 22.12 .2010 \\
\hline & covid19refusers.com & 24.5 .2020 \\
\hline
\end{tabular}

erklärt alles zusammen (und von jedem ein mehr oder weniger großer Anteil) das Geschehen recht gut. Für viele Menschen sind die Folgen der Reaktionen auf die Pandemie (Lockdown) - noch - deutlicher spürbar als die der Pandemie selber (das ändert sich allerdings zunehmend) und verursachen Stress durch existenzielle Bedrohungen, gegen die man sich hilflos fühlt. Die überall weitgehend unkontrollierte mediale Verbreitung von Falschmeldungen, die medialen Filterblasen (Menschen bekommen nur noch diejenigen Nachrichten, die zu ihrem Weltbild passen) und die in manchen Medien gleichsam „eingebaute“ Radikalisierung und Hass-Sprache verstärken, ebenso wie eine bestimmte genetische Prädisposition, dieses Erleben und den begleitenden Affekt der Angst. Dass Einsamkeit Stress verursacht und chronischer Stress die Immunabwehr schwächt, hatte sich zu Anfang der Pandemie noch nicht herumgesprochen [14-16].

Ein weiterer Gesichtspunkt erscheint mir in diesem Zusammenhang von Interesse und Bedeutung. Die weltgrößten IT-Unternehmen - Google, Facebook, Apple - versuchen zwar einerseits, Lügen und Fake-News bezüglich Covid-19 und der Pandemie von ihren eigenen Plattformen zu löschen. Dies darf jedoch andererseits nicht darüber hinwegtäuschen, dass sie aufgrund ihrer vielfältigen Aktivitäten die Verbreitung solcher Inhalte durch Online-Dienste, Tools und Computerprogramme überhaupt erst ermöglichen, die zu ihrem eigentlichen Geschäftsmodell gehören. In einer neuen Studie mit dem Titel „Profiting from the Pan- demic“ untersuchten Wissenschaftler vom Oxford Internet Institute an der dortigen Universität insgesamt 120 Websites, von denen jeweils 40 (a) Proteste gegen staatliche Einschränkungen (Verhaltensregeln, Ausgangsbeschränkungen, Lockdown, Shutdown), (b) betrügerische Aktivitäten (falsche Medikamente, erfundene Wohltätigkeitsinstitutionen zur Spendenannahme, funktionsunfähige Gesichtsmasken etc.) oder (c) falsche Informationen zum Coronavirus bzw. zur Corona-Pandemie verbreiteten [1]. Diese Webseiten haben klangvolle Namen, wie aus $>$ Tab. 1 ersichtlich ist. Manche von den 120 Webseiten existieren schon länger und wurden umetikettiert („repurposed“), die älteste von ihnen ging am 6. Juli 1995 online.

Die genannten Firmen (und noch viele andere, kleinere Firmen) bieten Dienste zur Entwicklung und Erstellung von Webseiten an (Web-Entwicklungs-Tools), stellen Speicherplatz und Verarbeitungskapazität (Webspace) zur Unterbringung (Hosting) von Websites auf ihren Webservern zur Verfügung (Webhosting) und halten entsprechende Software zur Auswertung und Vermarktung der auf den Webseiten gesammelten Daten vor (Stats-Tools, Adtracking). Bei einigen dieser Werkzeuge (Tools) handelt es sich um kostenlose „Widgets“, d. h. um kleine Computerprogramme, die es Webdesignern erlauben, die Möglichkeiten und Funktionalitäten ihrer Webseiten zu erweitern. Sie werden überall im Internet verwendet, oft ohne dass die Unternehmen, die sie herstellen, wissen, wer sie wozu einsetzt. Das ist bei Werkzeugen leider prinzi- 
piell so: Wer Hämmer produziert, hat keinen Einfluss darauf, ob jemand damit ein Haus baut oder jemanden erschlägt. Hierzu gehört beispielsweise Software zur Ermöglichung verschiedener Hardware- und Software-Komponenten (z. B. die Smartphones, Computer und Betriebssysteme verschiedener Hersteller) oder die Abwicklung finanzieller Transaktionen über Apple Pay durch die genannten Protest-, Betrugs- und Desinfomations-Webseiten. Die gleichen Top-Technologieunternehmen (Apple, Facebook, Google), deren Werkzeuge überall und von Kriminellen zu kriminellen Zwecken verwendet werden, verdienen zunächst einmal daran, denn all diese „IT Tools and Services “ werden ja verkauft. Zugleich geben diese Firmen aber auch seit Monaten vor (und reden viel darüber), dass sie Betrüger bekämpfen, die von der Pandemie profitieren wollen, und Desinformationen nach Kräften löschen.

Die Analyse von 40 Covid-Desinformationsseiten förderte fast 2000 Werkzeuge von Google, mehr als 800 von Facebook und mehr als 360 von Apple zutage. Die Firma Cloudflare, ein Anbieter von „Content-Delivery“ und Schutz vor Hacker-Angriffen, lieferte zudem fast 550 Komponenten auf den Desinformationsseiten, und die Firma Amazon hatte mehr als 330. Weil es keinen groBen Aufwand darstellt, die Werkzeuge von einem Anbieter durch die eines anderen bei der Gestaltung und Funktionalisierung von Webseiten zu ersetzen, kann nur eine konzertierte Anstrengung über Unternehmen, Länder und Gerichtsbarkeiten hinweg dieses Problem der kriminellen Anwendung von Werkzeugen lösen. „Bezüglich Protestseiten und Seiten mit angeblicher Desinformation unterstützen wir ein freies und offenes Internet. “ - So oder so ähnlich denken und reden die besagten Firmen (wenn sie sich überhaupt äußern). Und solange sie so gut mit diesen Werkzeugen verdienen, wird sich wahrscheinlich nur unter deutlich mehr Druck etwas ändern - man muss hier auf die EU hoffen und setzen, denn sonst ist ja niemand weit und breit erkennbar!

\section{Gedrückte Stimmung durch Corona}

Nach einer Anfang Dezember 2020 veröffentlichten Meinungsumfrage bewirkt die Corona-Pandemie bei Leistungsträgern im

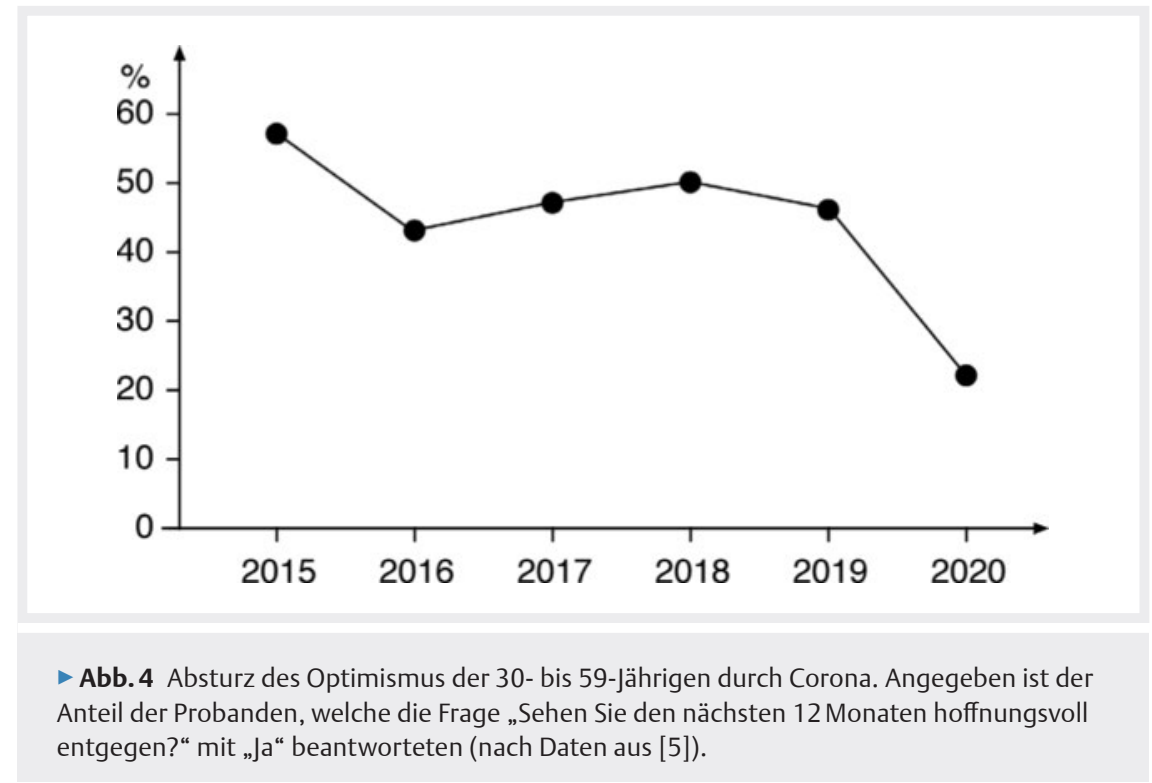

mittleren Alter (30-59 Jahre) eine Verunsicherung mit nur wenig Hoffnung auf eine bessere Zukunft. Der Gesamtverband der Deutschen Versicherungswirtschaft (GDV) hatte die Studie beim Allensbach-Institut für Demoskopie in Auftrag gegeben. Befragt wurden dabei 1047 Teilnehmer im Alter von 30-59 Jahren. Diese Gruppe, die oft auch als „Generation Mitte“ bezeichnet wird, stellt $70 \%$ der Erwerbstätigen und erwirtschaftet über $80 \%$ der steuerpflichtigen Einkünfte. Sie stellt damit das Rückgrat unserer Gesellschaft dar, und genau deswegen muss man sich darüber Sorgen machen, dass in genau dieser Gruppe der Menschen Angst und Verunsicherung deutlich zunehmen ( $\triangleright$ Abb.4); Optimismus? - Fehlanzeige! [5, 20, 27].

Demnach sehen nur noch weniger als ein Viertel der Befragten (22\%) den kommenden Monaten hoffnungsvoll entgegen, wohingegen dies im letzten Jahr noch $46 \%$ angaben. Zudem fühle sich jeder Zweite $(48 \%)$ schlechter als vor der Krise. Am schlimmsten sei die Tatsache, dass man nicht absehen könne, wie lange die Pandemie dauert. Angst vor einer Ansteckung hätten etwa $41 \%$. Die Mehrheit der Befragten gibt an, das Klima in der Gesellschaft habe sich stark in die falsche Richtung verändert - mit einer Zunahme von Ängsten, Verunsicherung und Aggressivität. Dass jeder Zweite einen verstärkten Rückzug der Menschen ins Private wahrnimmt und viele Dinge, die für selbstverständlich erachtet worden waren, nun besser wertgeschätzt werden, kann man positiv bewerten. Aber fast jeder Zweite macht sich große Sorgen um die Auswirkungen auf die deutsche Wirtschaft und weitere $26 \%$ machen sich sogar sehr große Sorgen.

Bedingt durch die vermehrte Angst, Verunsicherung und Vereinsamung hat auch die Inanspruchnahme der Telefonseelsorge deutlich zugenommen. Seit einem Vortrag vor etwa 400 ehrenamtlich in der Telefonseelsorge tätigen Personen weiß ich, dass Einsamkeit der Hauptgrund für einen Anruf bei der Telefonseelsorge ist. Es kam dort im Laufe der Corona-Pandemie zu einem deutlichen Anstieg der Anrufe um fast $50 \%$. In nahezu allen Gesprächen sei Corona das Hauptthema gewesen, mittlerweile überwiegen jedoch wieder die Klagen über Einsamkeit. Das wurde durch das Weihnachtsfest nicht besser - haben doch einsame Menschen die größten Schwierigkeiten mit dem Fest der Liebe und der Familie. Wie eng doch Viren und Weihnachten zusammenhängen. Unsere Welt ist komplex. Corona macht vor allem ihre Schwächen deutlich. Hoffentlich lernen wir daraus.

\section{Interessenkonflikt}

Es gibt keinen Interessenkonflikt. 


\section{Korrespondenzadresse}

Prof. Dr. Dr. Manfred Spitzer

Universität Ulm

Abteilung für Psychiatrie

Leimgrubenweg 12-14

89075 Ulm, Deutschland

Literatur

[1] Au Y, Howard PN, Project Ainita. Profiting from the Pandemic. Moderating COVID-19 Lockdown Protest, Scam, and Health Disinformation Websites. Oxford Internet Institute, 2.12.2020 https:// comprop.oii.ox.ac.uk/research/posts/ profiting-from-the-pandemic/; abgerufen am 15.12.2020

[2] Carfi A, Bernabei R, Landi F. Persistent Symptoms in Patients After Acute COVID-19. JAMA 2020; 324: 603-605

[3] Deisenhammer F, Borena W, Bauer A, et al. 6-month SARS-CoV-2 antibody persistency in a Tyrolian COVID-19 cohort. Wien Klin Wochenschr 2006. doi: 10.1007/s00508020-01795-7

[4] Fox SE, Akmatbekov A, Harbert JL, et al. Pulmonary and cardiac pathology in African American patients with COVID-19: an autopsy series from New Orleans. Lancet Respir Med 2020. doi.org/10.1016/S22132600(20)30243-5

[5] GDV - Gesamtverband der Deutschen Versicherungswirtschaft. Die Generation Mitte im Corona-Jahr 2020. Monitorstudie des Instituts für Demoskopie Allensbach im Auftrag des Gesamtverbands der Deutschen Versicherungswirtschaft, GDV https://www. gdv.de/resource/blob/64586/4047494c2077b273000a02eab8ef956b/generation-mitte-2020 praesentation-data.pdf; abgerufen am 6.12.2020

[6] Koopmans PC, Bakhtali R, Katan AA, et al. Return to work following sickness absence due to infectious mononucleosis. Occupational Medicine 2010; 60: 249-254

[7] Ledford H. US authorization of covid vaccine marks new phase in safety monitoring. Nature 2020; 588: 377-378

[8] Oxley TJ, Mocco J, Majidi S, et al. LargeVessel Stroke as a Presenting Feature of Covid-19 in the Young. NEJM 2020. doi: 10.1056/NEJMc2009787

[9] Pardi N, Hogan MJ, Porter FW, et al. mRNA vaccines - a new era in vaccinology. Nature Reviews Drug Discovery 2018; 17: 261-279

[10] Polack FP, Thomas SJ, Kitchin N, et al. Safety and Efficacy of the BNT162b2 mRNA Covid-19 Vaccine. N Engl J Med 2020. doi: 10.1056/NEJMoa2034577
[11] Rhea EM, Logsdon AF, Hansen KM, et al. The $\mathrm{S} 1$ protein of SARS-CoV-2 crosses the blood-brain barrier in mice. Nature Neuroscience 2020 . doi.org/10.1038/s41593020-00771-8; abgerufen am 17.12.2020

[12] Servick K. For survivors of severe COVID-19, beating the virus is just the beginning. Science 8.4.2020 https://www.sciencemag. org/news/2020/04/survivors-severe-covid19-beating-virus-just-beginning; abgerufen am 19.12.2020

[13] Spitzer M. Pandemie! Und wer geht hin? Nervenheilkunde 2009; 28: 505-508

[14] Spitzer M. Pandemie. Was die Krise mit uns macht und was wir aus ihr machen. München: mvg Verlag; 2020

[15] Spitzer M. Psychologie und Pandemie. Die Auswirkungen des Corona-Virus auf den Einzelnen und auf die Gesellschaft. Nervenheilkunde 2020; 39: 274-283

[16] Spitzer M. Pandemie - historisch, systematisch, kulturell und (sozial-) politisch. Nervenheilkunde 2020; 39: 363-372

[17] Spitzer M. Corona-Kollateralschäden: Informationen, Demonstrationen und Operationen. Nervenheilkunde 2020; 39: 444-451

[18] Spitzer M. Gesichtsmasken im Unterricht. Vor- und Nachteile der Bedeckung der unteren Gesichtshälfte in Zeiten der Corona-Pandemie. Nervenheilkunde 2020; 39: $522-532$

[19] Solomon IH, Normandin E, Bhattacharyya $S$, et al. Neuropathological features of Covid-19. NEJM 2020. doi.org/10.1056/ NEJMc2019373

[20] Starzmann P. Coronakrise schlägt der „Generation Mitte“ kräftig aufs Gemüt. Der Tagesspiegel, 2.12.2020 www. tagesspiegel.de/politik/jeder-zweite-fuehlt -sich-heute-schlechter-coronakrise-schlaegt -der-generation-mitte-kraeftig-aufsgemuet/26679388.html; abgerufen am 6.12 .2020

[21] Taquet M, Luciano S, Geddes JR, et al. Bidirectional associations between COVID-19 and psychiatric disorder: retrospective cohort studies of 62354 COVID-19 cases in the USA. Lancet Psychiatry 2020. doi. org/10.1016/S2215-0366(20)30462-4

[22] Timberg C. Covid disinformation sites often use tools from Google, Facebook and Apple, report finds. The Washington Post 4.12.2020 https://www.washing tonpost.com/technology/2020/12/04/ covid-scam-disinformation/; abgerufen am 5.12.2020

[23] Townsend L, Dyer AH, Jones K, et al. Persistent fatigue following SARS-CoV-2 infection is common and independent of severity of initial infection. PLoS ONE 2020; 15 (11): e0240784

[24] vfa. Die forschenden Pharma-Unternehmen. Impfstoffe zum Schutz vor der Coronavirus-Infektion Covid-19, 19.12.2020 https://www.vfa.de/de/ arzneimittel-forschung/woran-wirforschen/impfstoffe-zum-schutz-vorcoronavirus-2019-ncov; abgerufen am 19.12.2020

[25] Wajnberg A, Amanat F, Firpo A, et al. Robust neutralizing antibodies to SARS-CoV-2 infection persist for months. Science 2000; 370: $1227-1230$

[26] White PD, Thomas JM, Kangro $\mathrm{H}$, et al. Predictions and associations of fatigue syndromes and mood disorders that occur after infectious mononucleosis. Lancet 2001; 358: 1946-1953

[27] Wolf V. „Generation Mitte“ im Stimmungstief. Studie des Instituts für Demoskopie Allensbach im Auftrag des Gesamtverbands der Deutschen Versicherungswirtschaft (GDV). Pro Christliches Medienmagazin, 4.12.2020 https://www. pro-medienmagazin.de/gesellschaft/ gesellschaft/2020/12/04/allensbach-studie -generation-mitte-im-stimmungstief/; abgerufen am 6.12.2020

[28] Wolff JA, Malone RW, Williams P, et al. Direct gene transfer into mouse muscle in vivo. Science 247: 2019; 1465-1468

[29] Dennis A et al. Multi-organ impairment in low- 1 risk individuals with long COVID. medRxiv preprint, posted October 16, 2020. doi.org/10.1101/2020.10.14.202125 55; abgerufen am 18.11.2020

[30] Zhou X, Berglund P, Rhodes G, et al. Self-replicating Semliki Forest virus RNA as recombinant vaccine. Vaccine 1994; 12 : 1510-1514

Bibliografie

DOI https://doi.org/10.1055/a-1246-0550 Nervenheilkunde 2021; 40: 62-68 (c) 2021. Thieme. All rights reserved. Georg Thieme Verlag KG, Rüdigerstraße 14, 70469 Stuttgart, Germany ISSN 0722-1541 